OPEN ACCESS

Edited by:

Marco António Campinho, Centro de Ciências do Mar (CCMAR),

Portugal

Reviewed by:

Veerle M. Darras,

KU Leuven, Belgium

Paul Webb,

California Institute for Regenerative

Medicine, United States

*Correspondence:

S. Randal Voss

srvoss@uky.edu

Specialty section: This article was submitted to Thyroid Endocrinology, a section of the journal

Frontiers in Endocrinology

Received: 20 January 2019 Accepted: 25 March 2019

Published: 12 April 2019

Citation:

Crowner A, Khatri S, Blichmann D and Voss SR (2019) Rediscovering the

Axolotl as a Model for Thyroid Hormone Dependent Development.

Front. Endocrinol. 10:237.

doi: 10.3389/fendo.2019.00237

\section{Rediscovering the Axolotl as a Model for Thyroid Hormone Dependent Development}

\author{
Anne Crowner, Shivam Khatri, Dana Blichmann and S. Randal Voss* \\ Department of Neuroscience, Spinal Cord and Brain Injury Research Center, and Ambystoma Genetic Stock Center, \\ University of Kentucky, Lexington, KY, United States
}

The Mexican axolotl (Ambystoma mexicanum) is an important model organism in biomedical research. Much current attention is focused on the axolotl's amazing ability to regenerate tissues and whole organs after injury. However, not forgotten is the axolotl's equally amazing ability to thwart aspects of tissue maturation and retain juvenile morphology into the adult phase of life. Unlike close tiger salamander relatives that undergo a thyroid hormone regulated metamorphosis, the axolotl does not typically undergo a metamorphosis. Instead, the axolotl exhibits a paedomorphic mode of development that enables a completely aquatic life cycle. The evolution of paedomorphosis allowed axolotls to exploit relatively permanent habitats in Mexico, and preadapted axolotls for domestication and laboratory study. In this perspective, we first introduce the axolotl and the various meanings of paedomorphosis, and then stress the need to move beyond endocrinology-guided approaches to understand the axolotl's hypothyroid state. With the recent completion of the axolotl genome assembly and established methods to manipulate gene functions, the axolotl is poised to provide new insights about paedomorphosis and the role of thyroid hormone in development and evolution.

Keywords: axolotl, paedomorphosis, metamorphosis, thyroid hormone, ambystoma

\section{INTRODUCTION}

Mexican axolotls (Ambystoma mexicanum) have been studied in laboratories throughout the world for over two-hundred years (1). Beginning in the early nineteenth century, French expeditions to Mexico brought preserved adult specimens back to Paris for examination by curators at the Jardin des Plantes. Esteemed zoologist Georges Cuvier originally classified these specimens as larvae of an unknown species (2). It was not until decades later, when living axolotls were brought to Paris and a laboratory population was established by Auguste Duméril, that these presumptive larval forms were found to be reproductively mature and capable of metamorphosis. From the same axolotl spawn, Duméril (3) observed that most sibs reached an adult state and some reproduced while retaining larval characteristics including external gills, while a few individuals metamorphosed into forms typical of terrestrial salamanders (4). The observation of both metamorphic and nonmetamorphic forms arising from a single spawn inspired theories and experiments to explain the axolotl's unusual mode of paedomorphic development (5). While much has been learned from studies of the axolotl and other salamanders, the mechanistic basis of paedomorphosis remains largely unknown. 


\section{Paedomorphosis}

Paedomorphosis is a somewhat confusing term because it has been used to explain variation at evolutionary, ecological, and genetic levels of inquiry. At an evolutionary level, paedomorphosis is used to describe a specific pattern of developmental variation among ancestral and descendant species (6). The ancestral mode of development in salamanders is generally thought to include a single, obligate metamorphosis which partitions the life cycle between an early aquatic phase and a more terrestrial adult phase. Indeed, close tiger salamander (A. tigrinum) relatives of the axolotl are known to invariably undergo a metamorphosis (Figure 1). In contrast, the axolotl typically does not undergo a metamorphosis; axolotls are paedomorphic because they express ancestral juvenile traits in the adult stage of life. Paedomorphosis thus provides an evolutionary explanation for how new patterns of variation arise among species; the biphasic life cycle of an ancestral species was truncated somehow during evolution to yield a paedomorphic species. Within the lexicon of heterochrony, a theory that associates changes in developmental timing to the origin of new forms, Gould (6) proposed that paedomorphic salamanders arose during evolution as a result of changes in mechanisms that regulate metamorphic timing. While such description is useful for describing evolutionary patterns of developmental variation, what we ultimately seek is proximatelevel understanding of timing mechanisms that regulate the expression of paedomorphosis.

In ecological studies, paedomorphosis is used to describe patterns of developmental variation among individuals within species and populations. At this level, paedomorphosis is thought to be an adaptive developmental strategy for exploiting favorable larval habitats for growth and reproduction (8), although

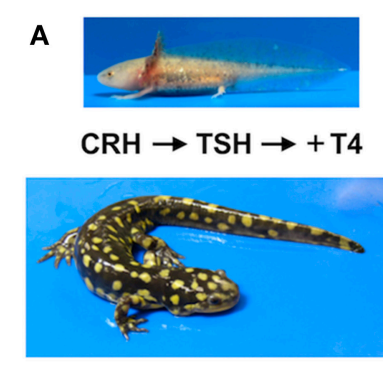

Metamorphosis
B

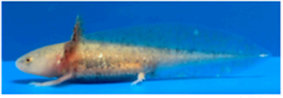

CRH ? TSH ? - T4

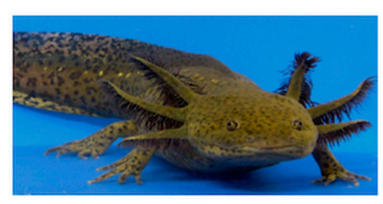

Paedomorphosis
FIGURE 1 | (A) The ancestral salamander mode of development is metamorphosis. Metamorphosis in the tiger salamander is regulated by the hypothalamus-pituitary-thyroid (HPT) axis. At a critical time during larval development, corticotrophin releasing hormone $(\mathrm{CRH})$ from the hypothalamus stimulates thyrotrophic cells in the pituitary to release thyroid stimulating hormone $(\mathrm{TSH})$, which in turn stimulates the thyroid gland to secrete thyroid hormone (TH). Increasing $\mathrm{TH}(+)$ triggers metamorphic changes in target cells. (B) The derived paedomorphic mode of development. Paedomorphosis in the axolotl results in the retention of ancestral larval characters into the adult phase of life. Although $\mathrm{CRH}$-like and TSH-like activity are present in the axolotl hypothalamus and pituitary respectively, and TSH and TH treatment can induce metamorphosis, the HPT axis is not activated during larval development. Modified from Johnson and Voss (7). non-adaptive variation in the expression of paedomorphosis is known (9). The mechanisms that allow for such plasticity are presumably influenced by an individual's genetic makeup, health status, and environmental cues. For example, studies of salamanders that express paedomorphosis facultatively have shown clear ecological correlates. Paedomorphosis is more frequent in permanent aquatic habitats that do not undergo seasonal drying $(10,11)$. It has been argued that ecological conditions largely dictate the expression of metamorphosis or paedomorphosis in facultative species to increase fitnessassociated traits, such as body size (bigger is generally better) and the probability of earlier and more frequent reproduction (12). It is important to point out that genetics also plays a role. Evidence for a population genetic component of variation has been shown in experiments that altered the heritability of paedomorphosis in A. talpoideum over several generations of selection (10). However, such studies do not provide resolution of genetic factors that regulate the expression of paedomorphosis.

Paedomorphosis has also been used to describe developmental variation among siblings within genetic crosses. Taking advantage of the recent evolution of paedomorphosis among tiger salamander complex species, interspecific genetic crosses have been performed in the laboratory to segregate metamorphic and paedomorphic modes of development and map the genomic location of genetic factors (13-18). These crosses have identified major effect quantitative trait loci (QTL) that regulate the timing of metamorphosis and expression of paedomorphosis. For example, second generation backcross individuals of $A$. mexicanum x A. tigrinum hybrid crosses that inherit axolotl alleles at the met1 QTL delay metamorphosis or express paedomorphosis. It is not clear if genetic factors identified from interspecific crosses regulate the expression of metamorphosis and paedomorphosis in natural populations. However, this classical genetic approach presents an unbiased method to identify candidate genes and associated mechanisms that may be operative in natural populations.

\section{Endocrinology of Paedomorphosis}

Over the last century, axolotl paedomorphosis has been the subject of a number of physiological studies. We refer readers to two relatively recent reviews of the literature pertaining to the endocrinology of axolotl paedomorphosis $(7,19)$. Our goal here is to briefly review salient features of axolotl hypothyroidism to provide context for identifying mechanisms that may regulate metamorphic timing and expression of paedomorphosis.

Thyroid hormones play a central role in regulating amphibian metamorphosis (Figure 1). Increasing titers of thyroid hormone during larval development are associated with tissue-specific changes that occur during metamorphosis. Thus, the timing of metamorphosis is potentially associated with a number of mechanisms that regulate $\mathrm{TH}$ : (1) the hypothalamus-pituitaryaxis that regulates $\mathrm{TH}$ synthesis and secretion from the thyroid gland, (2) TH transport and uptake within cells, (3) activation and inactivation of TH within cells, (4) binding of TH to steroid nuclear receptors in the nucleus, and (5) the interaction of $\mathrm{TH}$ and TR with protein complexes that regulate transcription. $\mathrm{TH}$ levels in the axolotl remain low throughout larval development 
and do not increase at the time metamorphosis occurs in related tiger salamanders. However, the axolotl is capable of initiating and completing metamorphosis when thyroid hormone and other endocrine factors of the HPT axis are administered. Collectively, these and other results suggest a defect in the regulation of the HPT axis, perhaps at the level of the hypothalamus or pituitary (19-24). Given the importance of thyroid hormone feedback on HPT axis maturation and control, axolotl paedomorphosis presents a conundrum-are $\mathrm{TH}$ levels inherently too low to support normal development and function of the hypothalamus and pituitary, and/or are these glands relatively insensitive to $\mathrm{TH}$ feedback?

One mechanism that has been advanced to explain axolotl paedomorphosis is a failure in hypothalamic stimulation of pituitary thyrotropes that secrete thyrotrophin (TSH) to regulate thyroid activity (19). In contrast to mammals, corticotropin $(\mathrm{CRH})$ and not thyrotropin releasing hormone (TRH) mediates release of TSH from the larval amphibian pituitary (25). CRH treatment of metamorphic tiger salamanders decreases the time to metamorphosis (26), consistent with thyrotrophic stimulation, while $\mathrm{CRH}$ treatment does not increase circulating T4 levels in the axolotl (24). While this result is consistent with a failure in hypothalamic stimulation of the pituitary, CRHR2 expression in axolotl thyrotropes appears to be normal. This highlights critical knowledge gaps in our understanding of axolotl paedomorphosis. Studies that have interrogated aspects of endocrine regulation have not rigorously controlled axolotl age, body size, or sex. Inducing metamorphosis by $\mathrm{TH}$ treatment of adult axolotls ignores early larval developmental windows within which the HPT axis matures and becomes operative. Also, studies have not assessed mechanisms of thyroid hormone regulation within all HPT axis tissues. For example, while it is clear that axolotls have functioning thyroid hormone receptors (27), the expression of TRs in HPT axis tissues has not been assessed. Also, while $\mathrm{CRH}$ is clearly essential for releasing TSH during amphibian metamorphosis, the secretion of $\mathrm{CRH}$ or $\mathrm{CRH}$-like peptides has not been shown in the axolotl (19). Finally, much of what we know about the endocrinology of amphibian metamorphosis comes from studies of anurans, not from studies of salamanders. These two amphibian groups diverged several hundreds of millions of years ago and salamander families are minimally 150 million years diverged. Thus, it is not clear that the anuran metamorphic knowledge base, or the relatively fewer insights gained among salamander species, can provide a framework to conceptualize the HPT axis in axolotls. While endocrinologyguided approaches have provided important insights, there is need to consider other avenues of reasoning in the study of axolotl paedomorphosis.

\section{Genetics of Paedomorphosis}

As was introduced above, genetic studies are beginning to resolve the location of genetic factors within the axolotl genome that regulate metamorphic timing and expression of paedomorphosis. Primarily, axolotls have been crossed to metamorphic tiger salamanders to segregate alleles that affect paedomorph expression and metamorphic timing, however we highlight a study (17) that crossed the axolotl to a paedomorphic relative to identify genetic factors associated with T4 sensitivity. In that study, second generation $A$. mexicanum/A. andersoni paedomorphic hybrids were created and administered $50 \mathrm{mM} \mathrm{T} 4$ at the time metamorphosis occurs in metamorphic tiger salamanders. Siblings exhibited tremendous variation in metamorphic timing (160-day range) and some individuals remained paedomorphic after 150 days of continuous $\mathrm{T} 4$ treatment. Genetic linkage mapping was then used to identify three moderate effect QTL (met1-3) that additively explained variation in metamorphic timing, including met 1 first identified in A. mexicanum/A. tigrinum hybrids. This study showed that metamorphic timing is associated with QTL that segregate allelic variation for responsiveness to T4.

At the time these QTL were identified and in lieu of a sequenced axolotl genome, comparative genome mapping was used to identify candidate genes for met1-3. The expressed sequence tag (EST) that was used to initially map met1 showed similarity to nerve growth factor receptor (ngfr). As more genes were mapped, it became clear that this $n g f r-$ like gene corresponded to nradd and met 1 located to a genomic region that was uniquely structured during vertebrate evolution. The genes in this region are found on separate chromosomes in all other vertebrates, including the newt (Notophthalamus viridescens) which is member of a different salamander family (28). Page et al. (18) speculated that this chromosomal fusion may have brought genes into linkage that are relevant for paedomorph expression in Ambystoma, because several of the genes have neurological functions. In addition to nradd, which is expressed in the mouse hypothalamus (29), ccm2, map $2 k 3$, and genes from the SmithMagenis syndrome region in the human genome associate with met1. ccm2 is associated with neurovascularization (30) while map $2 k 3$ was recently identified as a superior memory candidate gene in SuperAgers (31). Smith-Magenis syndrome is primarily attributed to deletion polymorphisms of rail (32), a dosage-sensitive transcription factor that regulates multiple functions, including embryonic neurodevelopment, neuronal differentiation, and circadian rhythm. Although rai locates outside the met 1 region, it is possible that long range enhancers for rail or other flanking genes may locate within the met 1 region (33). While no candidate genes were identified for met2, met3 is associated with poulf1, a transcription factor associated with combined pituitary hormone deficiency in humans. This deficiency is associated with incomplete secretion of pituitary hormones involved in regulating growth and development, but not reproduction. This makes poulf1 a good candidate for metamorphic regulation because metamorphic and paedomorphic ambystomatids do not show differences in reproductive potential, although paedomorphic species can reproduce multiple times annually while metamorphic species breed once annually (7).

\section{New Axolotl Genome Resources}

Five years ago, it was difficult if not impossible to pursue studies of axolotl candidate genes identified by comparative mapping. At that time, the large axolotl genome $(32 \mathrm{~Gb})$ had not been sequenced and thus it was difficult to develop molecular probes 
and investigate gene functions. However, in just the past couple of years, the axolotl genome has been incrementally sequenced and recently a chromosome-level genome assembly was completed (34). Now it is possible to comprehensively evaluate met1-3 genomic regions for candidate genes and test gene functions using genome-editing approaches and transgenics. In this section, we review new, critical resources to identify and test candidate genes for future studies of axolotl paedomorphosis.

It is important to note that the method used to assembly the axolotl genome simultaneously increased the resolution of candidate genes within the met1 genomic region (34). Forty-eight individuals from the meiotic mapping panel that was used to map met1 (16) were sequenced to $2 \mathrm{x}$ depth to identify polymorphisms that in turn were used to order genomic scaffolds into chromosomes by linkage analysis. The 48 individuals that were sequenced were not randomly drawn from the mapping panel. Instead, individuals that exhibited recombination within the met 1 genomic region were purposely chosen for sequencing to more finely resolve the boundaries of recombination that differentiate early (metamorphic) vs. late (paedomorphic) metamorphosing individuals. Inspection of these recombination boundaries against the background of the physically ordered loci yielded a high confidence genomic region of relatively few candidate genes (Figure 2).

The ordering of map2k3-nradd-and setd 2 is the same in the genome assembly as it was determined previously by genetic linkage analysis. Page et al. (18) mapped these loci near the maximum inflection point of the met 1 LOD peak, implicating them as especially good candidate genes. The new genome assembly revealed new, physically-linked candidates, including dhrs $7 b$, tmem11, and natd1 from the Smith-Magenis syndrome region, and $k c n j 12, k l h l 18$, smarcc1, and three anonymous, predicted genes. Using BLASTn and BLASTx searchers of the anonymous genes against NCBI databases, we discovered the likely identity of all three loci: camp (LOC101951429), ccn1-like (LOC102363594), and c16orf89 (LOC102943813). While camp is known to be syntenic with setd2, kif9, and khlh18 in the human genome, ccnl-like and c16orf89 are not expected to map to the met1 region, which only contains loci from human chromosomes 3 and 17. $c c n 1$ is a member of the CCN gene family, which contains six different members (CCN1-6). CCN family members typically have an $\mathrm{N}$-terminal secretory signal peptide and four structural domains: an insulin-like growth factor binding protein-like domain, a von Willebrand factor type $\mathrm{C}$ repeat (VWC) domain, a thrombospondin-homology type 1 repeat (TSP1) domain, and a C-terminal cysteine-knotcontaining (CT) domain. Interestingly, the met1 ccn1-like gene shows sequence similarity to $c c n 1$ but has a 30 bp deletion in the hinge region between the VWC and TSP1 domains (Figure 2). We determined the location of each presumptive CCN ortholog in the axolotl genome assembly, including a second ccn1-like gene that showed higher sequence identity to $c c n 1$-vertebrate orthologs. The position of the second conl-like gene in the axolotl genome assembly suggests it to be the true vertebrate ccn 1 ortholog and thus the ccn1-like gene in the met1 region appears to represent a novel gene. An ancient origin for this novel gene seems likely because it is equally divergent in amino acid similarity from human, Xenopus tropicalis, and axolotl ccn 1 orthologs (Figure 2). In contrast, the positioning of c16orf89 in the met genome region likely reflects an intrachromosomal inversion as several genes linked to c16orf89 in the human genome are found on axolotl chromosome 2, although some distance away.

\section{How to Test the Candidate Genes for Paedomorphosis?}

It is important to point out that none of the candidate genes in the met1 region have been annotated to endocrine gland development or endocrine process gene ontologies (Table 1). Two genes (setd2, smarcc1) that regulate global patterns of transcription during early neural development in mammals maybe interesting candidates to pursue because brain transcriptional activity is generally lower in larval axolotls than larval tiger salamanders $(18,35)$. Thyroid hormone and NGF signaling (perhaps via nradd) mediate early neuronal development in mammals, and based upon transcriptional data, c16orf89 is predicted to play a role in thyroid gland development and function. Given the multifunctional roles of $\mathrm{CCN}$ family members, ccn1-like presents an attractive candidate. However, nothing is known about this genes function and none of the genes discussed above seem to standout above the rest. As a path forward, we believe that all of the genes in the met 1 region can be quickly tested for function using CRISPR-Cas9, which is known to efficiently knock-out genes in the axolotl. For example, CRISPR-Cas9 was used recently to create insertion/deletion polymorphisms in fat 3 to generate offspring that presented limb and kidney defect phenotypes seen in the short toes axolotl mutant (36). In a similar vein, CRISPR-Cas9 could be used as a strategy to test candidate genes in the met1 genomic region using offspring from axolotl $\mathrm{x}$ tiger salamander crosses. For example, it is well-established that all axolotl $\mathrm{x}$ tiger salamander F1 hybrids undergo metamorphosis. This suggests that dominant tiger salamander alleles at candidate genes are sufficient to induce metamorphosis. Conversely, this suggests that paedomorphosis is associated with the inheritance of recessive alleles that may be partially functional or null in regards to inducing metamorphosis. To test this hypothesis, it would be efficient to knockout candidate genes in F1 hybrids as only the tiger salamander allele would need to be edited. F1 individuals could be assayed for paedomorphosis or delayed metamorphic timing and thus in short order, each of the candidate genes could be tested functionally. We note that it would also be informative to knock out met1-3 candidate genes in other vertebrates, including frogs which do not present paedomorphosis.

\section{CONCLUSION}

Although the Mexican axolotl has been studied for over 150 years, the mechanism associated with its unique paedomorphic mode of development remains unknown. Endocrinology studies have established the importance of thyroid hormone in regulating amphibian metamorphosis. The axolotl does not show an increase in thyroid hormone during early development and thus 


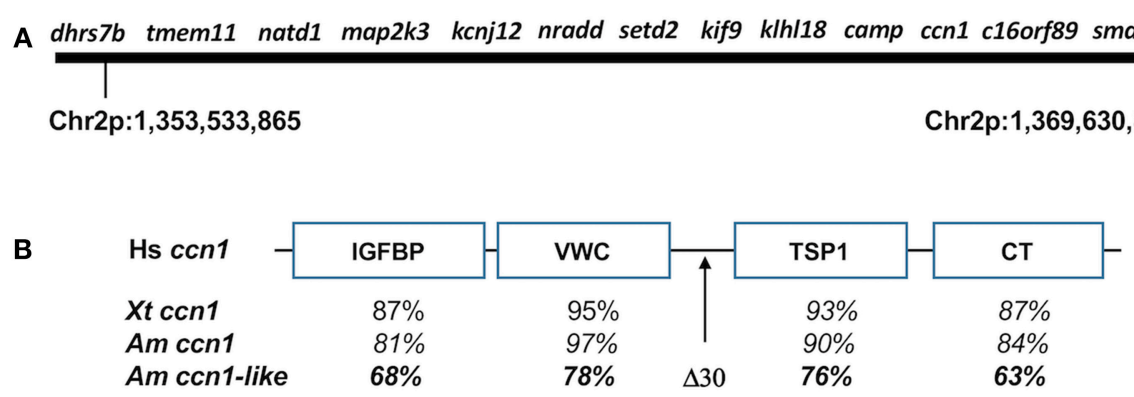

FIGURE 2 | (A) Map of genes from axolotl chromosome 2 (Chr2p) that associate with met1. (B) General structure of CCN family proteins showing insulin-like growth factor binding protein-like (IGFBP), a von Willebrand factor type C repeat (WWC), thrombospondin-homology type 1 repeat (TSP1), and a C-terminal cysteine-knot-containing (CT) domains. Human (Hs) ccn1 (GenBank: AAB84227.1) was used as a reference to compare domain-specific amino acid similarity among Xenopus tropicalis (Xp) ccn1 (GenBank: OCA36969.1), A. mexicanum ccn1 (AMEXTC_0340000257024_cysteine-rich), and A. mexicanum ccn1-like (AMEXTC_0340000025590_LOC102363594). The A. mexicanum ccn1-like sequence has a unique deletion of 30 amino acids between the WWC and TSP1 domains.

TABLE 1 | Candidate genes from the met1 region with functional information obtained from the NCBI Gene database.

\begin{tabular}{|c|c|}
\hline Gene ID & Gene information \\
\hline dhrs $7 b$ & Short-chain dehydrogenase/reductase family member. Possibly could function in steroid hormone regulation. \\
\hline tmem11 & Mitochondrial inner-membrane protein thought to regulate mitochondrial morphogenesis. \\
\hline natd1 & The function of this gene is unknown but transcripts in mice embryonic stem cells suggest a role in hematopoiesis. \\
\hline map2k3 & MAP kinase-mediated signaling cascade member that activates MAPK14/p38-MAPK in response to mitogens and environmental stress. \\
\hline kcnj12 & Inwardly rectifying K+ channel generally associated with heart function although broadly expressed among other tissues in human. \\
\hline nradd & Highly similarity to the p75 neurotrophin receptor ngfr that functions in neurotrophin signaling in rodents. \\
\hline setd2 & $\begin{array}{l}\text { Histone methyltransferase that is specific to lysine- } 36 \text { of histone } \mathrm{H} 3 \text {. Methylation of this residue is associated with active chromatin. Interacts with histone } \\
\mathrm{H} 2 \mathrm{~A} . \mathrm{z} \text { to regulate embryonic neurogenesis in mice. }\end{array}$ \\
\hline kif9 & Kinesin motor protein that functions in the regulation of spindle length and chromosome alignment during mitosis. \\
\hline klh/18 & Associates with cul3 ubiquitin ligase to regulate cell cycle entry. \\
\hline camp & An antimicrobial and immune response protein. \\
\hline ccn1-like & Novel CCN family gene. CCN family proteins regulate many cellular responses that are critical for skeletal, vascular, and neural development. \\
\hline c16orf89 & The function of this gene is unknown but human transcripts are enriched in the thyroid gland. \\
\hline smarcc1 & $\begin{array}{l}\text { Important component of the large ATP-dependent chromatin remodeling complex SNF/SWI which functions during brain development to regulate } \\
\text { transcription globally. }\end{array}$ \\
\hline
\end{tabular}

fails to undergo metamorphosis. While many aspects of the HPT axis seem to be functional in the axolotl, and peripheral tissues are responsive to thyroid hormone treatment, endocrinology-guided studies have not resolved the basis of paedomorphosis. We argue the need to test candidate genes from genetic studies of axolotl paedomorphosis using new genomic resources available to the community. In particular, the new axolotl genome assembly has resolved a short-list of candidate genes for the met1 genomic region that can be efficiently tested using CRISPR-Cas9 to knockout gene functions. The recent development of essential genetic and genomic tools for the axolotl brings us closer to identifying

\section{REFERENCES}

1. Reiß CL, Olsson L, Hoßfeld U. The history of the oldest self-sustaining laboratory animal: 150 years of axolotl research. J Exp Zool Part B. (2015) 324:393-404. doi: 10.1002/jez.b.22617

2. Cuvier G. Recherches anatomique sur les reptiles regardes encore comme douteux par les naturalistes; faites a l'occasion de l'axolotl, rapport_e par M. de Humboldt du Mexique. In: Humboldt A von, Bonpl A, editors. Voyage de mechanisms of paedomorphic development and understanding the role of thyroid hormone in development and evolution.

\section{AUTHOR CONTRIBUTIONS}

AC, SK, DB, and SRV collectively wrote the paper.

\section{FUNDING}

SRV was supported by the National Institutes of Health (P40OD019794, R24OD010435). Deuxieme partie. Tome 1. Recueil d'observations de zoologie et d'anatomie comparee. vol. 1. Paris: Schoell et Dufour (1811). p. 93-126.

3. Dumeril A. Reproduction, dans la menagerie des reptiles au Museum d'Histoire naturelle, des axolotls, batraciens, urodeles a branchies persistantes de Mexico (Siredon mexicanus, vel Humboldtii), qui n'avient encore jamais ete vus vivante en Europe. Compt Rend Hebd Seances Acad Sci. (1865a) 60:765-7. 
4. Dumeril A. Nouvelles observations sur les axolotls, batraciens urodeles de Mexico (Siredon mexicanus vel Humboldtii) nes dans la menagerie des reptiles au Mus_eum d'Histoire naturelle, et qui y subissent des metamorphose. Compt Rend Hebd Seances Acad Sci. (1865b) 61:775-8.

5. Kollman J. Das Ueberwintern von europäis-chen Frosch und Triton Iarven und die Umwand-lung des mexikanischen Axolotl. Verh Naturforsch Ges Basel. (1885) 7:387-98.

6. Gould SJ. Ontogeny and Phylogeny. Cambridge, MA: Belkap Press (1977).

7. Johnson CK, Voss SR. Salamander paedomorphosis: linking thyroid hormone to life history and life cycle evolution. Curr Top Dev Biol. (2013) 103:229-58. doi: 10.1016/B978-0-12-385979-2.00008-3

8. Wilbur HM, Collins JP. Ecological aspects of amphibian metamorphosis. Science. (1973) 182:1305-14. doi: 10.1126/science.182.4119.1305

9. Whiteman HH. Evolution of facultative paedomorphosis. Quart Rev Biol. (1994) 69:205-21. doi: 10.1086/418540

10. Semlitsch RD. Paedomorphosis in Ambystoma talpoideum: effects of density, food, and pond drying. Ecology. (1987) 68:994-1002. doi: 10.2307/1938370

11. Semlitsch RD, Harris RD, Wilbur HM. Paedomorphosis in Ambystoma talpoideum: maintenance of population variation and alternative life history pathways. Evolution. (1990) 44:1604-13.

12. Ryan TJ, Semlitsch RD. Growth and the expression of alternative life cycles in the salamander Ambystoma talpoideum (Caudata: Ambystomatidae). Biol J Linn Soc. (2003) 80:639-46. doi: 10.1111/j.1095-8312.2003.00260.x

13. Voss SR. Genetic basis of paedomorphosis in the axolotl, Ambystoma mexicanum: a test of the single gene hypothesis. J Hered. (1995) 86:441-7. doi: 10.1093/oxfordjournals.jhered.a111618

14. Voss SR, Shaffer HB. Adaptive evolution via a major gene effect: paedomorphosis in the Mexican axolotl. Proc Natl Acad Sci USA. (1997) 94:14185-9. doi: 10.1073/pnas.94.25.14185

15. Voss SR, Shaffer HB. Evolutionary genetics of metamorphic failure using wildcaught versus laboratory axolotls (Ambystoma mexicanum). Mol Ecol. (2000) 9:1401-8. doi: 10.1046/j.1365-294x.2000.01025.x

16. Voss SR, Smith JJ. Evolution of salamander life cycles: a major effect QTL contributes to both continuous and discrete variation for metamorphic timing. Genetics. (2005) 170:275-81. doi: 10.1534/genetics.104.0 38273

17. Voss SR, Kump KD, Walker JA, Shaffer HB, Voss GJ. Thyroid hormone responsive QTL and the evolution of paedomorphic salamanders. Heredity. (2012) 109:293-8. doi: 10.1038/hdy.2012.41

18. Page RB, Boley MA, Kump DK, Voss SR. Genomics of a metamorphic timing QTL: met1 maps to a unique genomic position and regulates morph and species-specific patterns of brain transcription. Genome Biol Evol. (2013) 5:1716-30. doi: 10.1093/gbe/evt123

19. De Groef B, Grommen SVH, Darras VM. Forever young: endocrinology of paedomorphosis in Mexican axolotl (Ambystoma mexicanum). Gen Comp Endocrinol. (2018) 266:194-201. doi: 10.1016/j.ygcen.201 8.05.016

20. Blount RF. The effects of heteroplastic hypophyseal grafts upon the axolotl, Ambystoma mexicanum. J Exp Zool A Ecol Genet Physiol. (1950) 113:717-39. doi: $10.1002 /$ jez.1401130312

21. Taurog A. Effect of TSH and long-acting thyroid stimulator on thyroid 131I-metabolism and metamorphosis of the Mexican axolotl (Ambystoma mexicanum). Gen Comp Endocrinol. (1974) 24:257-66. doi: 10.1016/0016-6480(74)90180-4

22. Jacobs GFM, Kühn ER. Thyroid function may be controlled by several hypothalamic factors in frogs and at least by one in the neotenic axolotl. In: Proceedings of the XIth International Symposium on Comparative Endocrinology, Malaga (1989), p.174.

23. Rosenkilde P, Ussing AP. What mechanisms control neoteny and regulate induced metamorphosis in urodeles? Int J Dev Biol. (1996) 40:665-73.
24. Kühn ER, De Groef B, Van der Geyten S, Darras VM. Corticotropin-releasing hormone-mediated metamorphosis in the neotenic axolotl Ambystoma mexicanum: synergistic involvement of thyroxine and corticoids on brain type II deiodinase. Gen Comp Endocrinol. (2005) 143:75-81. doi: 10.1016/j.ygcen.2005.02.022

25. Denver RJ, Licht P. Neuropeptide stimulation of thyrotropin secretion in the larval bullfrog: evidence for a common neuroregulator of thyroid and interrenal activity in metamorphosis. J Exp Zool. (1989) 252:101-4. doi: 10.1002/jez.1402520114

26. Boorse GC, Denver RJ. Acceleration of Ambystoma tigrinum metamorphosis by corticotropin-releasing hormone. J Exp Zool. (2002) 293:94-8. doi: 10.1002/jez.10115

27. Galton VA. Thyroid hormone receptors and iodothyronine deiodinases in the developing Mexican axolotl, Ambystoma mexicanum. Gen Comp Endocrinol. (1992) 85:62-70. doi: 10.1016/0016-6480(92)90172-G

28. Keinath MC, Voss SR, Tsonis PA, Smith JJ. A linkage map for the Newt Notophthalmus viridecens: insights in vertebrate genome and chromosome evolution. Dev Biol. (2017) 426:211-8. doi: 10.1016/j.ydbio.2016.05.027

29. Mercader JM, Lozano JJ, Sumoy L, Dierssen M, Visa J, Gratacòs M, et al. Hypothalamus transcriptome profile suggests an anorexia-cachexia syndrome in the anx/anx mouse model. Physiol Genom. (2008) 35:341-50. doi: 10.1152/physiolgenomics.90255.2008

30. Fischer A, Zalvide J, Faurobert E, Albiges-Rizo C, TournierLasserve E. Cerebral cavernous malformations: from $\mathrm{ccm}$ genes to endothelial cell homeostasis. Trends Mol Med. (2013) 19:302-8. doi: 10.1016/j.molmed.2013.02.004

31. Huentelman MJ, Piras IS, Siniard AL, De Both MD, Richholt RF, Balak $\mathrm{CD}$, et al. Association of map $2 k 3$ gene variants with super memory in superagers. Front Aging Neurosci. (2018) 29:155. doi: 10.3389/fnagi.2018. 00155

32. Vilboux T, Ciccone C, Blancato JK, Cox GF, Deshpande C, Introne WJ, et al. Molecular analysis of the retinoic acid induced 1 gene (RAI1) in patients with suspected smith-magenis syndrome without the 17p11.2 deletion. PLoS ONE. (2011) 6:e22861. doi: 10.1371/journal.pone.0022861

33. Yan J, Bi W, Lupski JR. Penetrance of craniofacial anomalies in mouse models of Smith-Magenis syndrome is modified by genomic sequence surrounding Rai1: not all null alleles are alike. Am J Hum Genet. (2007) 80:518-25. doi: $10.1086 / 512043$

34. Smith J, Timoshevskaya N, Timoshevskiy VA, Keinath MC, Hardy D, Voss SR. (2019) A chromosome-scale assembly of the enormous (32 Gb) axolotl genome. Genome Res. (2019) 29:317-24. doi: 10.1101/gr.241901.118

35. Page RB, Boley MA, Smith JJ, Putta S, Voss SR. Microarray analysis of a salamander hopeful monster reveals transcriptional signatures of paedomorphic brain development. BMC Evol Biol. (2010) 10:199. doi: 10.1186/1471-2148-10-199

36. Labianca C, Hardy DL, Rodgers AK, Mishra NA, Timoshevskaya NY, Parichy DM, et al. A PCR based assay to detect the short toes allele in Axolotls. Axolotl. (2018) 3:8-12.

Conflict of Interest Statement: The authors declare that the research was conducted in the absence of any commercial or financial relationships that could be construed as a potential conflict of interest.

Copyright (c) 2019 Crowner, Khatri, Blichmann and Voss. This is an open-access article distributed under the terms of the Creative Commons Attribution License (CC $B Y)$. The use, distribution or reproduction in other forums is permitted, provided the original author(s) and the copyright owner(s) are credited and that the original publication in this journal is cited, in accordance with accepted academic practice. No use, distribution or reproduction is permitted which does not comply with these terms. 